\title{
Early echocardiographic screening for subclinical myocardial dysfunction in children and adolescents with dyslipidemia: why and when?
}

\section{Key message}

Dyslipidemia contributes to early atherosclerosis, premature cardiovascular disease, and subclinical ventricular dysfunction in children. This paper highlights the need for echocardiographic evaluation for impaired diastolic function of both ventricles and narrowing of the aortic valve and sinus of Valsalva. Therefore, early echocardiographic screening of children with primary hyperlipidemia should be considered.

Dyslipidemia is closely related to cardiovascular disease (CVD) in adults and children and adolescents. As the prevalence of obesity and metabolic syndrome in children increases, the progression of CVD may occur at a younger age. In the issue of Clinical and Experimental Pediatrics, El-Koofy et al. ${ }^{1)}$ presented an association between primary dyslipidemia and myocardial dysfunction in early childhood. Primary dyslipidemia is mainly familial and manifests in childhood, and disease severity is usually worse than that of secondary dyslipidemia. Although the authors analyzed a small number of patients (25 patients), which limits the study's power, considering that primary dyslipidemia in children is a relatively rare condition, this analysis is still valuable. This study's main strength is that it stresses the importance of recognition and screening for myocardial function using early echocardiography in children and adolescents.

Universal screening for all children has emerged as a new concept over the last decade in contrast to the previous strategy of screening children and adolescents based on risk factors alone. ${ }^{2)}$ According to the clinical practice guidelines for dyslipidemia in Korean children and adolescents published in 2017,3$)$ in children aged 1-9 years with a family history of early coronary artery

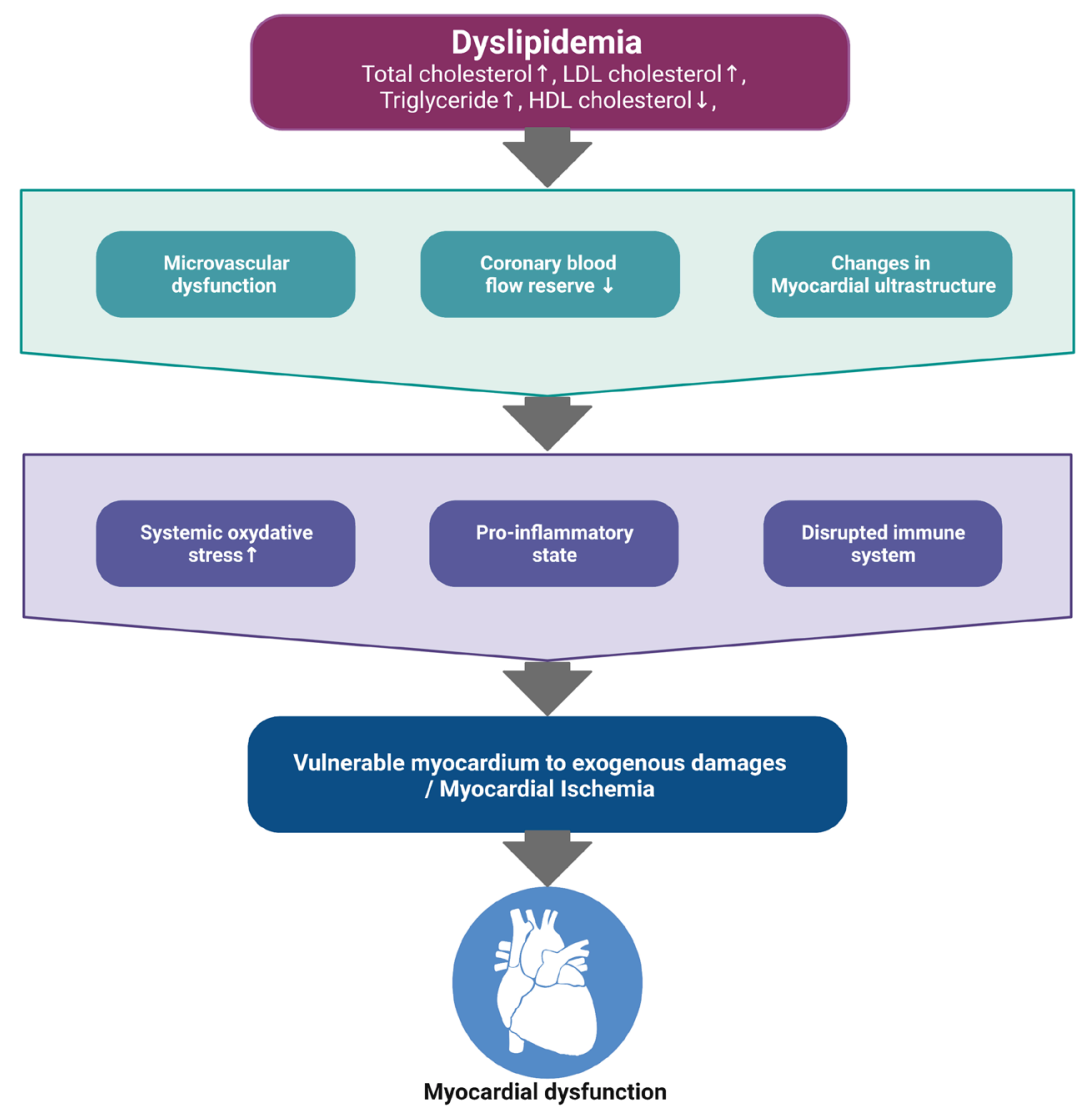

Graphical abstract. LDL, low-density lipoprotein; HDL, high-density lipoprotein. 
disease or dyslipidemia, screening for dyslipidemia using lipid profiles in a nonfasting state is recommended. The cholesterol level generally decreases by 10\%-20\% during puberty and increases again in the 20s. $\left.{ }^{4}\right)$ Therefore, considering this pattern, screening blood tests for dyslipidemia are required at 9-11 years of age and at 17-21 years for all children and adolescents, even if a clinical family history is lacking. ${ }^{3)}$ However, there are still no clear guidelines for the timing of screening for CVD using echocardiography. ${ }^{5)}$

Dyslipidemia contributes to early atherosclerosis and premature CVD. ${ }^{6,7)}$ It is known that increased total cholesterol, lowdensity lipoprotein cholesterol, and triglyceride levels and a decreased high-density lipoprotein cholesterol level are involved in the microvascular function that is correlated to myocardial function. ${ }^{8)}$ Hypercholesterolemia decreases coronary blood flow reserve, making the myocardium more vulnerable to exogenous damage such as hemodynamic overload and myocardial ischemia. $\left.{ }^{9}\right)$ Furthermore, hypercholesterolemia may also result in changes in the myocardial ultrastructure through several mechanisms, ultimately impairing ventricular function. In an experimental rat model, it led to systemic oxidative stress and a proinflammatory state. ${ }^{10)}$ It disrupts the immune system and induces the production of autoantibodies for $G$ protein-coupled receptors, increasing myocardial vulnerability and aggravating heart damage. ${ }^{11)}$ It also significantly decreased the expression of the cardiac autophagy markers. ${ }^{12}$ As reported in previous studies, further studies are needed that screen for myocardial function in children and adolescents with dyslipidemia.

A previous study highlighted the need for echocardiographic evaluation upon identifying impaired diastolic function of both ventricles and narrowing of the aortic valve and sinus of Valsalva. ${ }^{1)}$ The authors arrived at this conclusion by measuring the M-mode, 2-dimensional, pulse-wave, and color Doppler blood flow velocity measurements of the heart valves. In addition, functional parameters, including ejection fraction, peak systolic and early/late diastolic velocities, and global myocardial performance index, were measured. These parameters are commonly measured and used in the clinical setting. In addition, as previously reported, mechanisms of myocardial change, impaired diastolic function, and changes in heart valve size are possible consequences of dyslipidemia. ${ }^{13)}$ Although it is well known that dyslipidemia is associated with premature CVD and myocardial dysfunction, limited guidelines mention the need for echocardiography in children and adolescents with dyslipidemia. Echocardiography is still an advanced diagnostic modality in many countries, as its cost is high and specialists are required to perform it. Nevertheless, experts in the field should discuss the specific guidance for performing echocardiography and the parameters that must be obtained for children and adolescents with dyslipidemia.

Early recognition and intervention for dyslipidemia in child. ren and adolescents are essential for preventing severe CVD in adulthood. As recent evidence indicates that dyslipidemia leads to myocardial dysfunction, echocardiography screening for subclinical ventricular dysfunction in children with dyslipidemia should be considered. Early echocardiographic screening for CVD and myocardial dysfunction in children with dyslipidemia requires discussion and further study. Future studies will elucidate the timing of lipid-lowering agents that reverse early ventricular dysfunction, thus decreasing morbidity and mortality rates.

See the article "Early myocardial functional abnormalities in primary dyslipidemia: clinical and echocardiographic observations in young children from a highly consanguineous popula. tion" via https://doi.org/10.3345/cep.2021.00598.

\section{Hyun Gyung Lee, $\mathrm{MD}^{1}$, Hwa Jin Cho, MD, $\mathrm{PhD}^{2}$}

${ }^{1}$ Division of Pediatric Endocrinology, Department of Pediatrics, Chonnam National University Children's Hospital, Chonnam National University Medical School, Gwangju, Korea; ${ }^{2}$ Division of Pediatric Cardiology and Cardiac Critical Care, Department of Pediatrics, Chonnam National University Children's Hospital, Chonnam National University Medical School, Gwangju, Korea

Corresponding author: Hwa Jin Cho, $\mathrm{MD}, \mathrm{PhD}$

Division of Pediatric Cardiology and Cardiac Critical Care, Department of Pediatrics, Chonnam National University Children's Hospital, Chonnam National University Medical School, 42 Jebong-ro, Dong-gu, Gwangju 61469, Korea

凶Email:chhj98@gmail.com

https://orcid.org/0000-0002-2458-8529

\section{Footnote}

Conflicts of interest: No potential conflict of interest relevant to this article was reported.

Acknowledgments: The graphic abstract was edited by Biorender.

ORCID:

Hwa Jin Cho @ https://orcid.org/0000-0002-2458-8529

\section{References}

1. El-koofy NM, Fattouh AM, Ramadan A, Elmonem MA, Hamed DH. Early myocardial functional abnormalities in primary dyslipidemia: clinical and echocardiographic observations in young children from a highly consanguineous population. Clin Exp Pediatr 2022;65:410-6.

2. Lozano P, Henrikson NB, Morrison CC, Dunn J, Nguyen M, Blasi PR, et al. Lipid screening in childhood and adolescence for detection of multifactorial dyslipidemia: evidence report and systematic review for the US Preventive Services Task Force. JAMA 2016;316:634-44.

3. Lim JS, Kim EY, Kim JH, Yoo JH, Yi KH, Chae HW, et al. 2017 Clinical practice guidelines for dyslipidemia of Korean children and adolescents. Ann Pediatr Endocrinol Metab 2020;25:199-207.

4. Webber LS, Srinivasan SR, Wattigney WA, Berenson GS. Tracking of 
serum lipids and lipoproteins from childhood to adulthood. The Bogalusa Heart Study. Am J Epidemiol 1991;133:884-99.

5. Di Salvo G, D'Aiello AF, Castaldi B, Fadel B, Limongelli G, D'Andrea A, et al. Early left ventricular abnormalities in children with heterozygous familial hypercholesterolemia. J Am Soc Echocardiogr 2012;25:107582.

6. Expert Panel on Integrated Guidelines for Cardiovascular Health and Risk Reduction in Children and Adolescents; National Heart, Lung, and Blood Institute. Expert panel on integrated guidelines for cardiovascular health and risk reduction in children and adolescents: summary report. Pediatrics 2011;128 Suppl 5(Suppl 5):S213-56.

7. Grundy SM, Stone NJ, Bailey AL, Beam C, Birtcher KK, Blumenthal RS, et al. 2018 AHA/ACC/AACVPR/AAPA/ABC/ACPM/ADA/AGS/ $\mathrm{APhA} / \mathrm{ASPC} / \mathrm{NLA} / \mathrm{PCNA}$ guideline on the management of blood cholesterol: a report of the American College of Cardiology/American Heart Association Task Force on Clinical Practice Guidelines. Circulation 2019; 139:e1082-143.

8. Yao YS, Li TD, Zeng ZH. Mechanisms underlying direct actions of hyperlipidemia on myocardium: an updated review. Lipids Health Dis 2020;19:23.

9. Fauchier L, de Labriolle A. Cholesterol levels and cholesterol lowering in idiopathic dilated cardiomyopathy. Eur Heart J 2005;26:1931; author reply 1931-2.

10. Han Q, Yeung SC, Ip MSM, Mak JCW. Dysregulation of cardiac lipid parameters in high-fat high-cholesterol diet-induced rat model. Lipids Health Dis 2018;17:255.

11. Moretti S, et al. A mast cell-ILC2-Th9 pathway promotes lung inflammation in cystic fibrosis. Nat Commun 2017;8:14017.

12. Cheng Y, Zhu Y, Zhang J, Duan X, Zhang Y. Large accumulation of collagen and increased activation of mast cells in hearts of mice with hyperlipidemia. Arq Bras Cardiol 2017;109:404-9.

13. Vitarelli A, Martino F, Capotosto L, Martino E, Colantoni C, Ashurov $\mathrm{R}$, et al. Early myocardial deformation changes in hypercholesterolemic and obese children and adolescents: a 2D and 3D speckle tracking echocardiography study. Medicine (Baltimore) 2014;93:e71.

How to cite this article: Lee HG, Cho HJ. Early echocardiographic screening for subclinical myocardial dysfunction in children and adolescents with dyslipidemia: why and when? Clin Exp Pediatr 2022;65:398-400. https://doi.org/10.3345/ cep. 2022.00031 